Article

\title{
Information Energy Mimics $\Lambda$ and CDM
}

\author{
Michael Paul Gough \\ Department of Engineering and Design, University of Sussex, Brighton, BN1 9QT, UK. \\ E-Mail:m.p.gough@sussex.ac.uk
}

\begin{abstract}
Stellar heated gas and dust makes a significant entropic/information energy contribution to the universe. At temperatures $\sim 10^{7}$ the $\sim 10^{86}$ bits are equivalent to $\sim 10^{70} \mathrm{~J}$, equivalent to the energy equivalence of the universe's $\sim 10^{53} \mathrm{~kg}$ ordinary baryon matter. A survey of stellar mass density measurements shows this dark energy contribution has a constant energy density that effectively mimics a cosmological constant over the redshift range $z<1.35$. The measurable difference between this information energy and a true cosmological constant is small, with a maximum difference of $<2 \%$ in Hubble parameter at $z \sim 2$. As information energy is significant and co-located with hot baryons it produces gravitational effects that resemble dark matter. Information energy is shown to be consistent with the dark matter effects observed in clusters of colliding galaxies (e.g. Bullet Cluster), with dark matter location specified by baryon location and strongest in regions of highest luminosity / temperature. The dark matter fraction measured in galaxy surveys more closely fits an information energy explanation than the fraction expected in the standard $\Lambda \mathrm{CDM}$ model. Information energy provides a solution to the cosmological coincidence problem and also would allow the cosmological constant to take the preferred zero value.
\end{abstract}

Keywords: dark energy; dark matter; $\Lambda$ CDM model

\section{Introduction}

In the standard $\Lambda \mathrm{CDM}$ model ordinary baryonic matter comprises only $5 \%$ of the universe with much larger contributions from dark energy, $68 \%$, and dark matter, $27 \%$. The energy density of matter, decreasing with universe expansion, has fallen below the level of a near constant dark energy, to change earlier decelerating expansion into the observed accelerating expansion of the present epoch [1,2]. Although dark energy is generally assumed to be the cosmological constant it still has no accepted physical explanation for its value[3,4]. Observations of galaxy rotation rates, galaxy gravitational focusing, and clusters of colliding galaxies all require a significant unseen dark matter in addition to visible baryons. Candidates for dark matter particles include WIMPS, Axions, and others [5]. However, all attempts to directly detect dark matter particles have drawn a blank, and, in the process, severely limited any possible particle energy range [6,7]. A survey of the dark matter field [8] suggests that more progress might be achieved through additional astrophysical observations and new considerations.

Here we consider the possible role of information energy. Matter and information both have equivalent energies. While mass, $m$, is equivalent to $m c^{2}$ of energy, Landauer's principle states that erasure of a bit of information will release $\mathrm{k}_{B} T \ln (2)$ of energy $[9,10]$. Note that Landauer's principle has now been experimentally verified for both classical and quantum information [11-14].

The $1^{\text {st }}$ Friedman equation [15] describing the expansion of the universe includes a term for the equivalent matter energy density, but ignores any information energy density contribution. Information energy naturally varies over time depending on star and structure formation and is therefore more difficult to include in a general universe description. Previous estimates of information bit numbers and temperatures of various astrophysical phenomena [16-20] have shown that the strongest information energy contribution is presently made by the $\sim 10^{86}$ bits of stellar 
heated gas and dust at temperatures $10^{6}-10^{8}$. Information energy should be included in universe energy accounting since $10^{86}$ bits at a temperature of $10^{7}$ have the same equivalent energy, $10^{70} \mathrm{~J}$, as that of the $\sim 10^{53} \mathrm{~kg}$ of all universe baryons.

We show below that information energy might provide a single explanation for the many effects hitherto attributed separately to the dark energy and dark matter phenomena.

\section{Information energy presently mimics a cosmological constant.}

As the information energy of stellar heated gas and dust is significant, we need to identify its previous variation over time. For simplicity, we make two assumptions. Firstly, we assume that the total bit number, $N$, of any large co-moving volume is governed by the Holographic Principle [21-23], and varies as $a^{2}$, where $a$ is the universe scale size, $a=1 /(1+z)$, where $z$ is the redshift. Secondly, we assume that, within any sufficiently large volume, the average temperature, $T$, representative of the stellar heated gas and dust varies in proportion to the fraction of baryons that have formed stars.

In figure 1 we plot a survey of measured stellar mass densities per co-moving volume as a function of scale size, a. The filled symbols [24-40] correspond to data compiled for a recent survey of stellar formation measurements (Table 2 of [41]). A subset of these data was already included in previous work [18-20], and open symbols [42-53] correspond to other measurements used in that previous work.

There is a clear growth in star formation with approximately one half of today's stars formed before redshift, $z=1.3$, and $25 \%$ formed after $z=0.7$. Despite considerable scatter in measured values in Fig.1, upper plot, there appears to be a significant change around redshift, $z \sim 1.35$ from a steep gradient in the past to a weaker gradient in recent times. Fitting straight line power laws (red lines, in Fig.1) to data points either side of $z=1.35$, we find power law fits of $a^{+1.08 \pm 0.16}$ for $z<1.35$, and $a^{+3.46 \pm 0.23}$, for $z>1.35$. Then we can assume average baryon temperature, $T$, is proportional to the fraction of baryons in stars and thus also varied as $a^{+1.08 \pm 0.16}$ for $z<1.35$. Thus the total stellar heated gas and dust information energy ( $\alpha N T$ ) varied as $a^{+3.08 \pm 0.16}$, corresponding to near constant energy density, or an equation of state parameter, value $w=-1.03 \pm 0.05$. In comparison, total information energy in the earlier period, $z>1.35$, varied as $a^{+5.46 \pm 0.23}$, corresponding to a phantom energy with $w=-1.82 \pm 0.08$.

We see that the information energy of stellar heated gas and dust in the recent period, $z<1.35$, could then explain dark energy, since $T$ closely follows the $a^{+1}$ gradient that would lead to information energy varying as $a^{+3}$, to effectively emulate a cosmological constant ( $w=-1$, blue line in Fig.1, upper plot). Thus information energy can account for dark energy quantitatively, both the present energy density value, and the recent period of near constant overall energy density. 


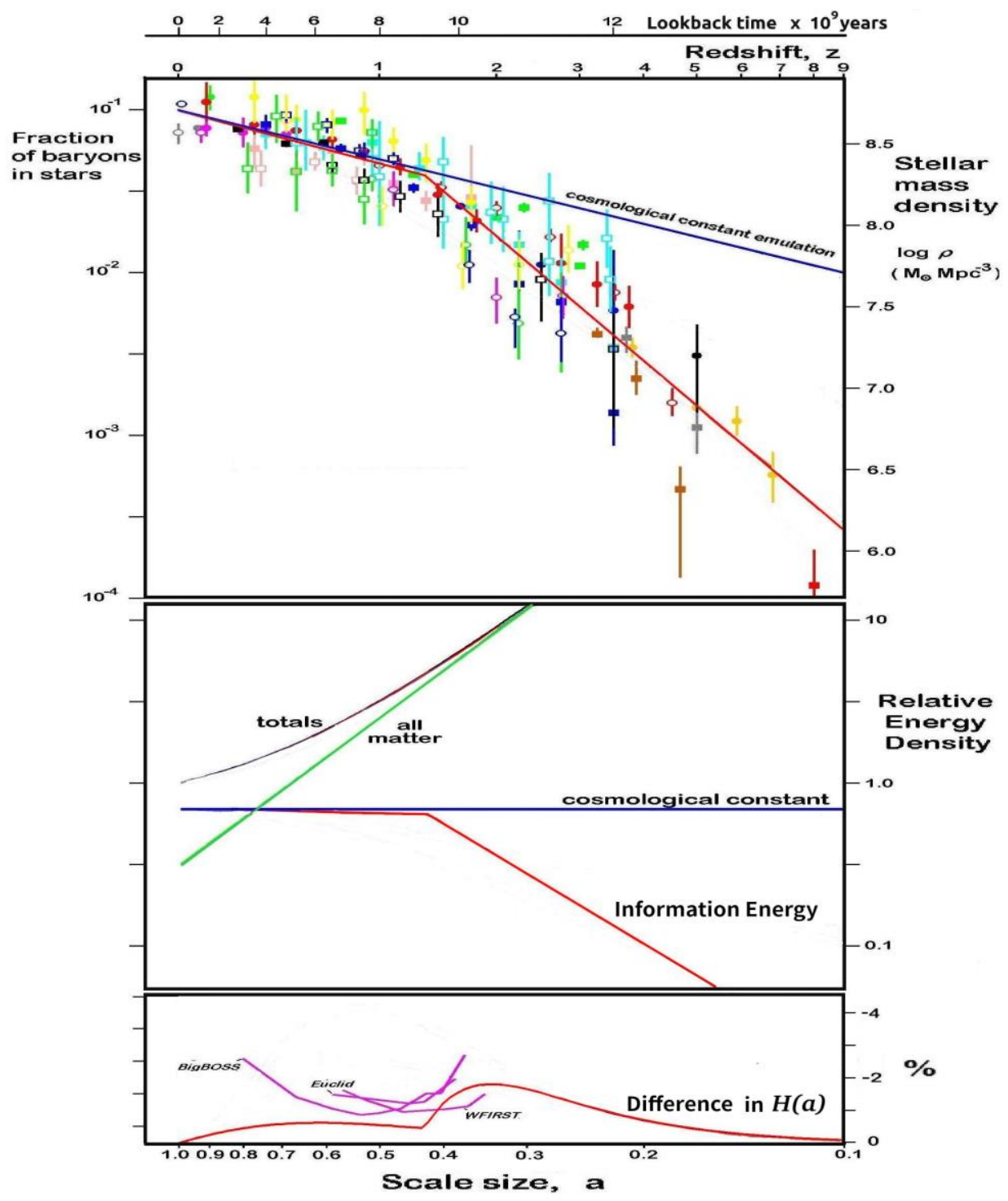

Figure 1. Upper Plot. Review of stellar mass density measurements for co-moving volumes as a function of universe scale size, $a$. Plotted lines: two red straight lines, power law fits $a^{+1.08 \pm 0.16, \quad(w=}$ $-1.03 \pm 0.05)$ for $z<1.35$, and $a^{+3.46 \pm 0.23},(w=-1.82 \pm 0.08)$ for $z>1.35$; blue line, the variation that would be required for information energy to fully emulate a cosmological constant over all scale sizes. Source references. Filled symbols: grey circle[24]; dark green circle[25]; magenta circle[26]; pink square[27]; red circle[28]; cyan square[29]; blue square[30]; yellow circle[31]; black square[32]; green square[33]; blue circle[34]; dark green square[35]; brown square[36]; orange circle[37]; grey square[38]; black circle[39]; red square[40]. Open symbols: grey circle[42]; dark green circle[43]; magenta circle[44]; pink square[45]; red circle[46]; cyan square[47]; blue square[48]; yellow circle[49]; black square[50]; green square[51]; blue circle[52]; dark green square[53].

Middle Plot. Relative energy densities, relative to total today(=1.0) for a cosmological constant, information energy, and all matter. The totals for all matter+cosmological constant, and for all matter+information energy are also shown.

Lower Plot. The difference in Hubble parameter, $H(a)$ to be expected for an information energy source of dark energy relative to that due to a cosmological constant, and compared to the resolutions of future missions: Euclid, BigBoss, and WFIRST[54-56]. 
We saw that information energy and matter equivalent energy were both similar $\sim 10^{70} \mathrm{~J}$ and information energy density today could easily equal the cosmological constant. In Fig.1. middle plot, we set the values today to be equal and then wish to see if the difference in expected time histories is detectable. We see that the total of information energy + all matter is practically indistinguishable from the total of a cosmological constant + all matter. At earlier times, the difference between dark energy sources is greater but the contributions to the total are masked by the much larger matter contribution at those times. So in Fig 1, lower plot, we emphasize the difference by plotting the relative difference in expected Hubble parameter, $H(a)$, values between information energy and cosmological constant sources of dark energy. We note that this difference is small, $<2 \%$, and close to, or even beyond, the resolution of some future dark energy measurements.

\section{Constant information energy density: natural feedback and/or limitation?}

The advent of accelerating expansion has been associated $[57,58]$ with directly causing a general reduction in galaxy merging, and structure formation. This reduction is evident in Fig.1 as the clear stellar mass density gradient change from $a^{+3.46 \pm 0.23}$ to $a^{+1.08 \pm 0.16}$. Once information energy was strong enough to initiate acceleration, this in turn inhibited star formation and consequently limited the growth of information energy itself. In our information energy explanation, this feedback mechanism should naturally cap the star formation gradient around a stable value $\sim a^{+1}$, constraining energy density to a constant value around the observed dark energy density to effectively mimic a cosmological constant. It also explains the timing of the change in stellar formation rate around $z \sim 1.35$, the power law value after $z \sim 1.35$, and the present ratio of dark energy to matter energy.

Algorithmic information theory provides a simple, independent, supporting argument for a constant information energy density related to star formation and accelerated expansion. The algorithmic information content of a system is given by the length of the shortest string of bits that can describe the position and momentum of all particles in the system [59]. This length is often called the algorithmic complexity or Kolmogorov complexity. Algorithmic information provides insights into the physics, as it has some features in common with thermodynamic entropy, including the requirement to adhere to the $2^{\text {nd }}$ law.

Stars form from clumps in molecular clouds, and especially from clumps in giant molecular clouds. In order to see how star formation has affected the universe algorithmic information content, we can simply consider changes in the universe averaged number of bits required per baryon per dimensional parameter. We must first decide on the level of resolution, or graining, required. For the purposes of our argument, we consider the two limiting resolutions: Planck length $\left(1.6 \times 10^{-35} \mathrm{~m}\right)$, the smallest dimension with any physical meaning; and Fermi length $\left(10^{-15} \mathrm{~m}\right)$, typical of nucleon dimensions and thus the largest to still describe particle interactions. Intergalactic baryons, bounded by the universe $\left(\sim 10^{27} \mathrm{~m}\right)$ require $\sim 205$ bits/parameter at Planck resolution, since the required accuracy is one part in $6 \times 10^{61}$ or one part in $2^{205}$. Similarly, baryons constrained within giant molecular clouds $\left(\sim 10^{18} \mathrm{~m}\right)$ require $\sim 175$ bits/parameter, and baryons in a typical star (e.g. the sun $10^{9} \mathrm{~m}$ ) require $\sim 145$ bits/parameter. At Fermi resolution the three equivalent values are each 65.7 bits/parameter less.

We assume a simple model based on the growth in the measured fraction of baryons that have formed stars up to the present value of $10 \%$ of all baryons (Fig.1). While the bits/parameter of the intergalactic baryons increase by one bit per universe doubling in size, those baryons within giant molecular clouds loose 30 bits per parameter when they form stars. Then Fig. 2. shows the resulting average bits/parameter/baryon using the growth history of star formation given by the observations summarised in Fig 1 (red lines). For comparison Fig. 2. also shows the variation if there was no star formation. The 30 bits lost by the present $10 \%$ of baryons that have formed stars has made a 
contribution of $\sim-3$ bits to the average while between $a=\sim 0.25$ and the present $a=1.0$ the remaining $90 \%$ of baryons have contributed $\sim+2$ bits to the average. The resulting overall average fall of $\sim 1$ bit/parameter/baryon is clearly independent of resolution between the limits of Planck and Fermi lengths, and implies a significant decrease in the total algorithmic information content of the universe, contrary to the $2^{\text {nd }}$ law.

Fortunately, the present dark energy density has additionally increased the total universe energy density by $\sim x 4$, resulting in acceleration that doubled the Hubble parameter and hence the size of the universe. The resulting accelerating expansion has provided the $90 \%$ intergalactic baryons, and effectively the universe baryon average, with one extra bit/parameter to counteract the reduction of one bit/parameter in algorithmic information due to star formation (horizontal dashed line of Fig.2). Then accelerating expansion appears to be required to ensure the universe's algorithmic information complies with the $2^{\text {nd }}$ law, supporting our suggestion of a direct link between star formation, information and acceleration.

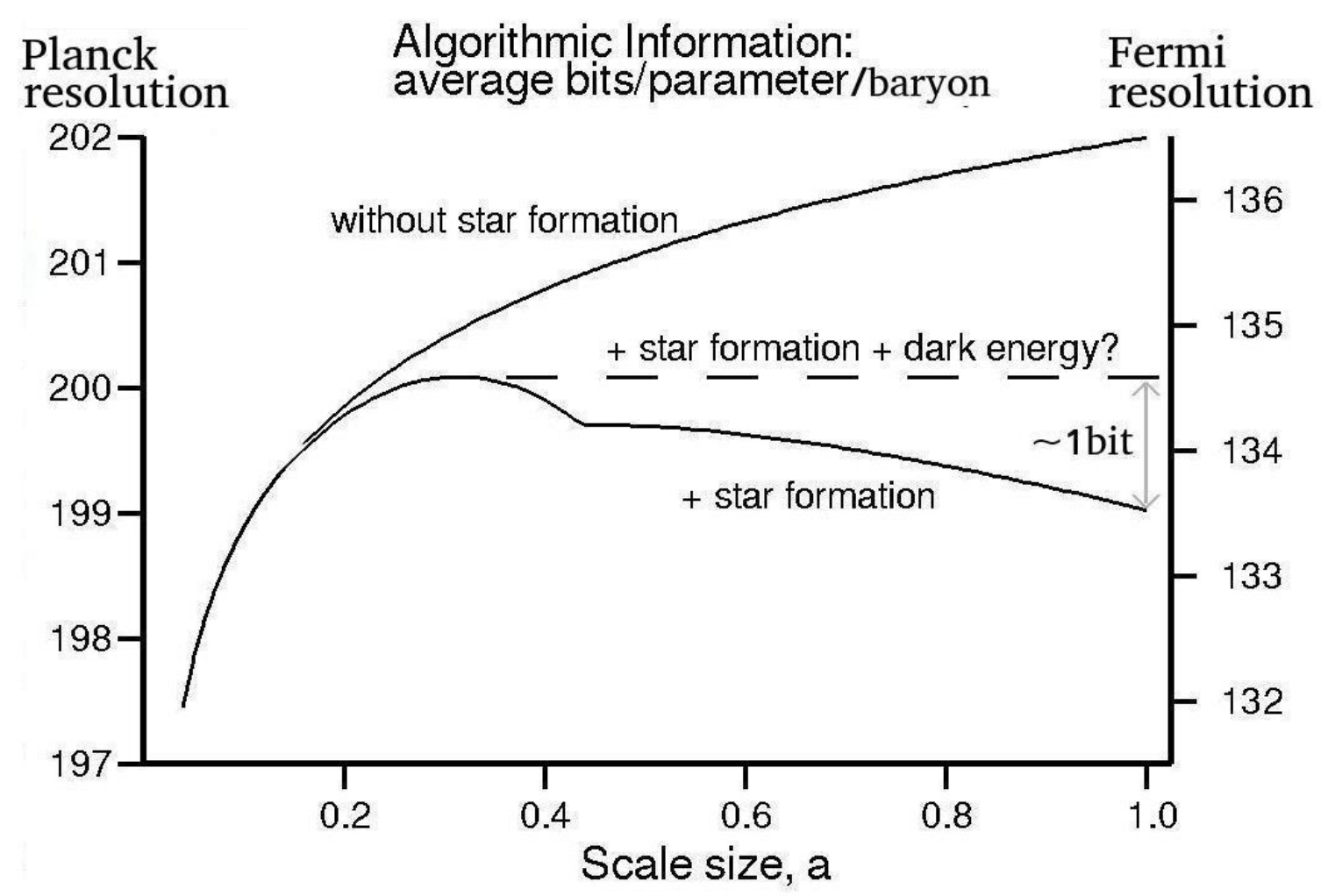

Figure 2. The average number of bits per parameter per baryon, averaged over all universe baryons, needed for an algorithmic information description.

\section{Phantom Information Energy at earlier times.}

The results of experiments to measure dark energy equation of state, $w$, usually assume a simple shape for the $\mathrm{w}(\mathrm{a})$ timeline, using a minimum number of parameters with a present value $w_{0}$, and the value at much earlier times $w_{0}+w_{\text {a }}$. Most astrophysical datasets, including Planck data [60-63], have been analysed to deduce cosmological parameters using the simple two parameter 'CPL' form of parameterisation [64] given by $w(\mathrm{a})=w_{\mathrm{o}}+(1-\mathrm{a}) w_{\mathrm{a}}$. This parameterisation assumes a smooth, continuous variation of $\mathrm{w}(\mathrm{a})$ from $w_{\mathrm{o}}+w_{\mathrm{a}}$ at very early times, $\mathrm{a}<<1$, through to $w_{\text {o }}$ today $(\mathrm{a}=1)$. The 2013, 2015, and 2018 Planck data releases,[60-63], include several dataset combinations where Planck data have been combined with other types of measurement and analysed using the CPL 
parameterisation, Fig.3. While it is usually noted that the resultant likelihood plots in $w_{\mathrm{o}}-w_{\text {a }}$ space include the case of a cosmological constant $\left(w_{0}=-1, w_{\mathrm{a}}=0\right)$ and therefore consistent with $\Lambda \mathrm{CDM}$, there is an overall bias towards a phantom dark energy with a significant negative range in $w$ a. The closest CPL parameter description for information energy, $w_{0}=-1.03, w_{\mathrm{a}}=-0.8$, is shown in Fig.3 to also lie near to the centre of the maximum likelihood region. Note information energy exhibits a much sharper transition than can be fully described by CPL (see Fig.1).

There is a mounting body of evidence to suggest that the dark energy density is dynamic, with a phantom dark energy $\left(w_{a}<0\right)$ at earlier times, compatible with an information energy source. Planck $\mathrm{CMB}$ data is not strongly constrained without combining with other data sets. On its own this Planck data yields $\mathrm{w}=-1.54,+0.62 /-0.50$ corresponding to a $\sim 2$ o shift into the phantom regime [61], effectively averaging over the whole range $0<z<1100$. Various combinations of other measurements have found a similar shift into the phantom regime [65-69]. An earlier phantom energy might also explain the problem of reconciling Hubble constant values found by different measurement techniques. This difference, or 'Hubble Tension', of $\sim 4.40$, is primarily between late universe higher values and the early universe Planck CMB lower value[70]. This tension could be explained by dynamic dark energy that was phantom at earlier times.
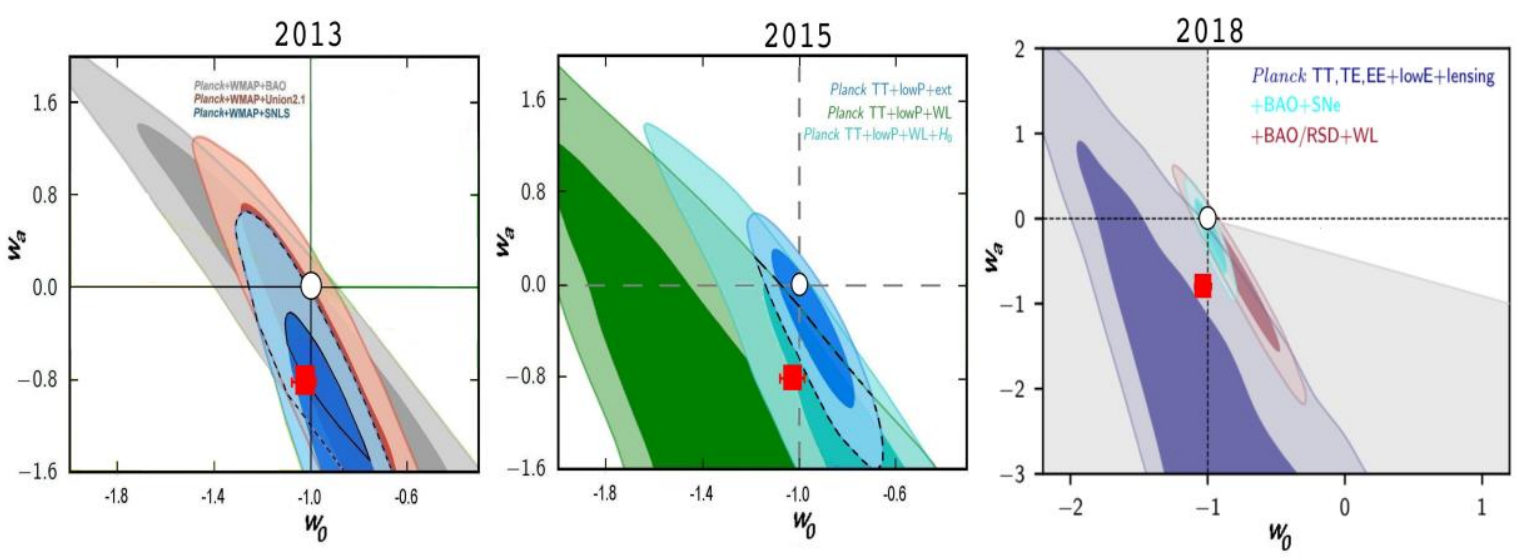

Figure.3. Information energy predictions compared with Planck dataset combination results of 2013, 2105, and 2018. 2D marginalised posterior distributions are shown by the $68 \%$ and $95 \%$ likelihood contours as a function of $w_{0}$ and $w_{\text {a }}$ for different dataset combinations (see [60-63] for dataset details). Symbols: white circle, cosmological constant; red square, information energy.

2013: The areas bounded by the black dashed line and the black continuous line correspond to the $95 \%$ and $68 \%$ likelihoods, respectively, that are common to all three datasets.

2015: Dashed line: 95\% common likelihood.

2018: The unshaded region is the only region that does not correspond to a phantom dark energy. 


\section{Information energy mimics dark matter}

Information energy is naturally located where baryons occur at high temperature and density and where information energy densities should be high enough to add significantly to baryon distortions of space-time. While the above dark energy effects of information energy are universe-wide, the dark matter effects will be different for each astrophysical object, and therefore more difficult to take into account in a quantitative manner.

The information energy explanation leads us to expect that the location of hot baryons will fully specify where dark matter attributed effects are strongest. Indeed, a high correlation has been found $[71,72]$ between the observed galaxy radial acceleration and that predicted from baryons, based on a total of 240 galaxies of various morphologies: 153 late-type galaxies, 25 early- type galaxies and 62 dwarf spheroidals. There is very little scatter and this strong empirical relation shows all galaxies studied follow the same radial acceleration relation, showing the dark matter contribution to be fully specified by the baryons. Thus dark and baryonic masses exhibit a strong coupling that is difficult for the $\Lambda \mathrm{CDM}$ model to explain, but follows directly from information energy.

Clusters of colliding galaxies are considered to provide some of the strongest evidence for the existence of dark matter. Optical observations show stars pass through the collision largely unhindered whereas X-ray observations show the galactic gas clouds, containing the majority of baryons, collide, slowing down or even halting. The location of dark matter is then identified from lensing measurements [73-75]. A study of the Bullet cluster [73], and of a further 72 mergers [74], both major and minor, finds no evidence for dark matter deceleration, with the dark mass remaining closely co-located with the stars and structure. Thus dark matter is found to be not concentrated around the baryon centre of gravity in the galactic gas clouds, and an upper limit is placed on any dark matter self-interaction. Clearly information energy could equally explain the dark matter effects, if the dominant contribution to information energy in these cases were from stellar heated gas and dust that generally passes with the stars straight through.

Observations of clusters of galaxies [76] show that the brightest galaxies are almost always found in the middle of those locations where gravitational lensing indicates the dark matter contribution is maximum. Clearly, this property is also consistent with an information energy explanation since information energy is proportional to temperature.

Information energy also fits with the favoured bottom up hierarchical structure formation with smaller objects forming first and effectively promoting the formation of larger structures, resembling $\mathrm{CDM}$ rather than hot dark matter. In the $\Lambda \mathrm{CDM}$ model gravitational lensing effects are due to higher densities of dark matter which have led to increased structure formation and brighter galaxies at those locations. As galaxies increase in brightness with increasing temperatures and higher entropies, the higher information energy densities should lead to both the observed gravitational lensing dark matter-like effects and further increases in structure formation.

In our information energy explanation, the present universe wide dark energy fraction of all energy $f_{D E} \sim 68 \%$ required to explain the universe expansion history is then equivalent to a similar universe-wide average dark matter fraction of all matter, $f_{D M} \sim 68 \%$. This value is significantly lower than the $f_{D M} \sim 85 \%$ of the Planck $\Lambda$ CDM model. It is beyond the scope of this work to survey all measured $f_{D M}$ values of astrophysical objects, ranging from globular clusters containing little dark matter to dwarf galaxies dominated by dark matter. However, a survey [77] of $1.7 \times 10^{5}$ massive early-type galaxies $z<0.33$ yields $f_{D M}=53 \%-72 \%$ within those galaxies' effective radius (radius that defines the sphere responsible for one half of the galaxy's light emission). Another survey of 584 typical star-forming galaxies, $z=0.8-1.0$ [78] finds $f_{D M}=65 \pm 12 \%$. Note the present information energy value $\sim 68 \%$ lies in the middle of both survey ranges while $\Lambda$ CDM $f_{D M} \sim 85 \%$ lies outside. 


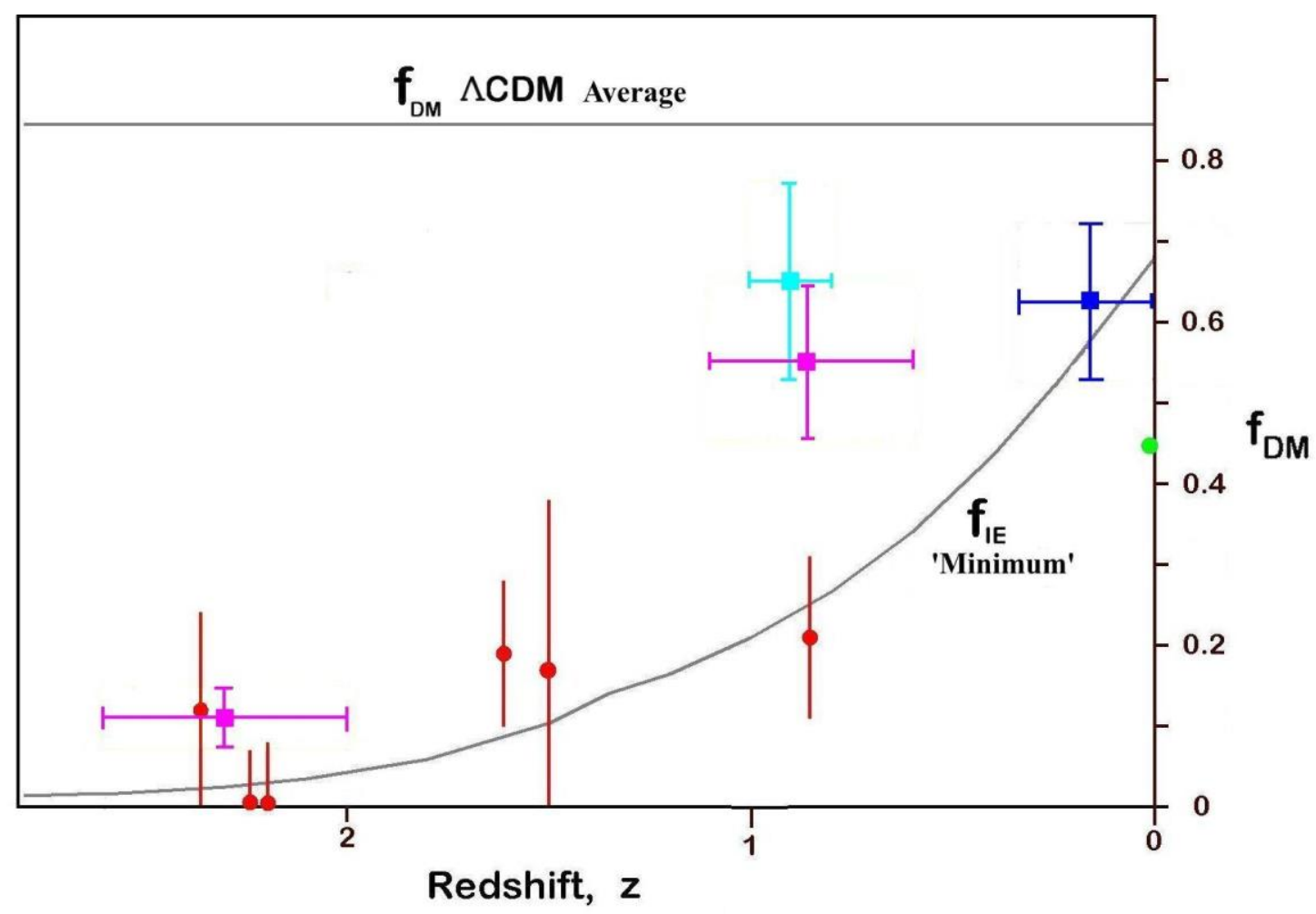

Figure 4. Observed $f_{D M}$ compared with average $\Lambda C D M f_{D M}$ and the information energy $f_{I E}$. 'minimum'. Survey results, square symbols: Dark Blue, $1.7 \times 10^{5}$ massive early-type galaxies $\mathrm{z}<0.33$ [77], Light Blue, 584 typical star-forming galaxies, $\mathrm{z}=0.8-1.0$ [78], Purple, 92 star forming galaxies, $\mathrm{z}=2.0-2.6$ and 106 star forming galaxies, $\mathrm{z}=0.6-1.1$ [79]. Individual galaxies, circle symbols: Red, six early star forming galaxies [79]; Green, Milky Way galaxy [80]. The fIE curve is calculated from the power law fits to the star formation data of Fig.1, assuming present $f_{I E}=68 \%$.

While values of $f_{I E}$ should be high in the high temperature baryon objects that we can observe, universe average $f_{I E}$ would have been much lower at earlier times when only a small fraction of baryons had formed stars. Therefore, we expect observable object $f_{D M}$ values at or above the universe average $f_{I E}$ value at that redshift ( $f_{I E}$ effectively provides a minimum value). In contrast, universe average $\Lambda C D M f_{D M}$ should have remained constant, independent of redshift, and observed object $f_{D M}$ values should be found distributed approximately evenly about $f_{D M} \sim 85 \%$. Fig.4. compares these universe average values with several galaxy surveys [77-79] and with six early star forming disk galaxies [79]. The variation in the dark matter contribution illustrated in Fig.4 emphasizes the relative absence of dark matter in early massive star forming galaxies of 10 billion years ago. Note surveys of early star forming galaxies made with the same instruments and analysis techniques, but in two different redshift ranges [79] (purple squares in Fig.4) show that galaxies at $z=2.0-2.6$ clearly have lower $f_{D M}$ values than those at $\mathrm{z}=0.6-1.1$. Overall, both survey and individually measured values of $f_{D M}$ are more consistent with the time varying minimum predicted by the information energy model than with the fixed $85 \%$ average value of the $\Lambda \mathrm{CDM}$ model.

An information energy explanation would explain how the early galaxies with little dark matter grew into the dark matter dominated galaxies of today. The spatial distributions of some galaxies and galaxy clusters have been found to exhibit an "assembly bias" [81]. The way in which those galaxies interact with their dark matter environments appears to be determined not just by their masses but also by their past formation history. This could also be consistent with an information energy explanation as information/entropy is a result of not just present processes but also the result of the past history of physical processes that operated on baryons. 
The milky way, Andromeda, and Centaurus-A galaxies have a number of satellite dwarf galaxies that orbit in the same plane with the majority co-rotating $[82,83]$. This observation is difficult to reconcile with $\Lambda \mathrm{CDM}$ as dark matter should be distributed in a sphere around the parent galaxy with satellite galaxies randomly distributed. However, this observation may be consistent with information energy as dark matter like effects should follow the location of the parent galaxy's hot baryons.

\section{Discussion and Summary}

All of the effects attributed to both dark energy and dark matter only occur through the action of gravitational forces. There is no evidence of dark energy or dark matter interacting with ordinary baryonic matter or photons through any of the other fundamental forces. This similarity argues for a common explanation, and information energy provides an attractive solution.

The information energy approach allowed us to quantitatively account for the present dark energy density value with proven physics, using the experimentally proven Landauer's Principle with realistic universe entropy estimates. Combining star formation measurements with the Holographic principle leads to the present near constant information energy density. The Holographic principle is generally accepted for black holes at the holographic bound, but remains only a conjecture for universal application[23].

The information energy explanation for dark energy allows the cosmological constant to take the more likely zero value [84]. Nevertheless, there is an interesting similarity between $\Lambda$ and information energy. $\Lambda$ has a characteristic energy with present value $\Delta \Lambda \sim 3 \times 10^{-3} \mathrm{eV}$, too small to relate to any relevant particle physics [85]. The form of equation for $\Delta \Lambda$ has been shown $[86,87$ ] to be directly equivalent to $\mathrm{k}_{B} T$, corresponding to the equivalent energy of a bit of information at $T \sim 35$. This low value is effectively the average baryon temperature if there was no star or structure formation, while here we have concentrated on the information associated with the much higher temperatures of stellar heated gas and dust, $T \sim 10^{7}$.

Information energy could also solve the "why now?" cosmological coincidence problem. Star formation had to have advanced sufficiently before information energy was strong enough to affect universe expansion. Star formation had also to have advanced sufficiently for the likelihood of intelligent beings evolving to observe an accelerating expanding universe. It is therefore not such a coincidence that we are around when dark energy and matter have similar order of magnitude energy densities.

Many effects previously attributed to dark matter: galaxy spin anomalies; gravitational lensing; lensing of clusters of colliding galaxies; and galaxy 'assembly bias' could all be caused by information energy. Information energy is consistent with the dark matter effects observed to be fully specified by baryon location, strongest at highest luminosity locations, consistent with measured dark matter fractions in galaxy surveys, and possibly also consistent with the observed alignment of satellite galaxies. This explanation for dark matter attributed effects is attractive as it does not require invoking new, exotic, and difficult to detect dark matter particles, nor does it require the new physics of MOND[88] and dark fluid theories[89].

Finally, the information energy explanation reduces a problem of two unexplained phenomena to a single phenomenon, providing an explanation emphasizing the two preferred requirements: 'simplicity' (wielding Occam's razor); and 'naturalness' (relying on mostly proven physics) [8,90], with a strong dependence on empirical data. 
Acknowledgements: The author is grateful for an Emeritus Professorship from the

University of Sussex.

\section{References.}

1. Riess, A.G.; Filippenko, A.V.; Challis, P.; Clocchiatti, A.; et al., Observational evidence from supernovae for an accelerating universe and a cosmological constant. Astron. J., 1998, 116, 1009-1038.

2. Perlmutter, S.; Aldering, G.; Goldhaber, G.; Knop, R.A.; et al. Measurements of $\Omega$ and $\Lambda$ from 42 high-redshift supernovae. Astrophys. J., 1999, 517, 565-586.

3. Carroll, S.M. Why is the Universe Accelerating? In Measuring and Modeling the Universe; Carnegie Observatories Astrophysics Series, Vol2; Freedmann, W.L., Ed.; Cambridge Univ Pr. Cambridge, UK, 2003.

4. Frieman, J.A.; Turner, M.S.; Huterer, D. Dark energy and the accelerating universe. Ann.Rev.Astron. Astrophys., 2008, 46, 385-432.

5. Pretzl, K. Dark Matter Searches, Space Science Reviews, 2007, 130, 63-72.

6. Bauer, D. , Buckley, J. , Cahill-Rowley, M., et al, Dark Matter in the Coming Decade: Complementary Paths to Discovery and Beyond, Physics of the Dark Universe, 2015, 7-8, pp 16-23.

7. Bertone,G., Hooper,D., Silk,J., Particle dark matter: Evidence, candidates and constraints, Physics Reports, 2005, 405,(5-6), 279-390.

8. Bertone, G., A new era in the search for dark matter, Nature, 2018, 562, 51-56.

9. Landauer, R.,Irreversibility and heat generation in the computing process. IBM J. Res.Dev. ,1961, 3, 183-191.

10. Landauer, R. Information is physical. Phys. Today, 1991, 44, 23-29.

11. Toyabe, S; Sagawa, T.; Ueda, M.; Muneyuki, E.; Sano, M. Experimental demonstration of information-to-energy conversion and validation of the generalized Jarzynski equality. Nat. Phys., 2010, 6, 988-992.

12. Berut, A.; Arakelyan, A.; Petrosyan, A.; Ciliberto, S.; Dillenschneider, R.; Lutz, E. Experimental verification of Landauer's principle linking information and thermodynamics. Nature, 2012, 483,187-189.

13. Jun,Y., Gavrilov, M.,Bechhoefer,J., High-Precision Test of Landauer's Principle in a Feedback Trap, Physical Review Letters, 2014, 113, 190601-1 to -5.

14. Yan,L.L., Xiong,T.P., Rehan,K., et al., Single_Atom Demonstration of the Quantum Landauer Principle, Phys. Rev. Lett.2018, 120, 210601.

15. Weinberg, A., Gravitation and Cosmology, Wiley, New York, 1972.

16. Frampton, P.H.; Hsu, S.D.H.; Kephart, T.W.; Reeb, D. What is the entropy of the universe? Class. Quant. Grav., 2009, 26, 145005, (7pp).

17. Egan, C.A.; Lineweaver, C.H. A larger estimate of the entropy of the universe. Astrophys. J., 2010, 710, 1825-1834.

18. Gough,M.P., Holographic Dark Information Energy, Entropy, 2011, 13, 924-935.

19. Gough, M.P., Holographic Dark Information Energy: Predicted Dark Energy Measurement, Entropy, 2013, 15, 1133-1149.

20. Gough,M.P.,A Dynamic Dark Information Energy Consistent with Planck Data, Entropy, 2014, 16, 1902-1916

21. T'Hooft, G. Obstacles on the way towards the quantization of space, time and matter- and possible solutions. Stud. Hist. Phil. Mod. Phys., 2001, 32, 157-180.

22. Susskind, L. The world as a hologram. J. Math. Phys., 1995, 36, 6377-6396.

23. Buosso, R. The holographic principle. Rev. Mod. Phys., 2002, 74, 825-874.

24. Li, C., White, S.D.M., The distribution of stellar mass in the low-redshift universe, Mon. Not. R. Astron. Soc., 2009, 398, 2177-2187.

25. Gallazzi,A., Brinchmann,J.,, Charlot,S., White,S.D.M., A census of metals and baryons in stars In the local universe, Mon. Not. R. Astron. Soc., 2008, 383, 1439-1458.

26. Moustakas J, Coil A, Aird J, et al., PRIMUS: constraints on star formation quenching and Galaxy merging and the evolution of the stellar mass function from $\mathrm{z}=0-1, A p$. J., 2013, 767:50 (34pp).

27. Bielby R, Hudelot P, McCracken HJ, et al., The WIRCam Deep Survey. I. Counts, colours, and mass-functions derived from near-infrared imaging in the CFHTLS deep fields, Astron. 
Astrophys. , 2012, 545, A23 (20pp).

28. Perez-Gonzalez PG, Rieke GH, Villar V, et al., The stellar mass assembly of galaxies from $\mathrm{Z}=0-4$ : analysis of a sample selected in the rest-frame near infrared with Spitzer, Ap. J., 2008, 675, 234-261.

29. Ilbert $\mathrm{O}, \mathrm{McC}$ racken $\mathrm{HJ}$, Le F 'evre $\mathrm{O}$, et al., Mass assembly in quiescent and star-forming Galaxies since z 4 from UltraVISTA, Astron. Astrophys. 2013, 556, A55(19pp).

30. Muzzin A, Marchesini D, Stefanon M, et al. The evolution of the stellar mass functions of star-forming and quiescent galaxies to $\mathrm{z}=4$ from the COSMOS/UltraVISTA survey, Ap. J. , 2013, 777, 18 (30pp).

31. Arnouts S, Walcher CJ, Le Fevre O, et al.,The SWIRE-VVDS-CFHTLS surveys: Stellar Assembly over the last 10Gyr., Astron. Astrophys. , 2007, 476, 137-150.

32. Pozzetti L, Bolzonella M, Zucca E, et al., zCOSMOS -10k bright spectroscopic sample. The bimodality in the galaxy stellar mass function, Astron. Astrophys. , 2010, 523, A13(23pp).

33. Kajisawa M, Ichikawa T, Tanaka I, et al. , MOIRCS deep survey IV evolution of galaxy stellar mass function back to z 3, Ap. J. , 2009, 702, 1393-1412.

34. Marchesini D, van Dokkum PG, Forster Schreiber NM, et al., The evolution of the stellar mass function of galaxies from $z=4$ and the first comprehensive analysis of its uncertainties, Ap. J., 2009, 701, 1765-1769.

35. Reddy NA, Dickinson M, Elbaz D, et al. GOODS-HERSCHEL measurements of the dust attenuation of typical star forming galaxies at high redshift, Ap. J., 2012, 744, 154(17pp).

36. Caputi,K.I,, Cirasuolo,M., Dunlop,J.S., et al. The stellar mass function of the most massive Galaxies at $3<\mathrm{z}<5$ in the UKIDSS Ultra Deep Survey, Mon. Not. R. Astron. Soc., 2011, $413,162-176$

37. Gonzalez V, Labbe I, Bouwens RJ, et al. , Evolution of galaxy stellar mass functions, mass densities, and mass-to light ratios from $\mathrm{z} 77$ to $\mathrm{z} 4$, Ap. J. Let., 2011, 735, L34(6pp).

38. Lee K-S, Ferguson HC, Wiklind T, et al., How do star-forming galaxies at $\mathrm{z}>3$ assemble their masses?, Ap. J., 2012, 752, 66(21pp).

39. Yabe K, Ohta K, Iwata I, et al. The stellar populations of Lyman break galaxies at $\mathrm{z} \sim 5$, Ap. J., 2009, 693, 507-533.

40. Labbe I, Oesch PA, Bouwens RJ, et al., The spectral energy distributions of $\mathrm{z} \sim 8$ galaxies from the IRAC ultra deep fields, Ap. J. Let., 2013, 777, L19(6pp).

41. Madau,P., Dickinson,M., Cosmic Star Formation History, Annual Review of Astronomy and Astrophysics, 2014, 52, 415-486.

42. Cole, S.; Peterson, B.A.; Jackson, C.; Peacock, J.A.; et al., The 2dF galaxy redshift survey., Mon. Not. R. Astron. Soc., 2001, 326, 255-273.

43. Dickinson, M.; Papovich, C.; Ferguson, H.C.; Budavari, T. The evolution of the global stellar mass density at $0<\mathrm{z}<3$. , Astrophys. J., 2003, 587, 25-40.

44. Rudnick, G.; Rix, H.W.; Franx, M.; Labbe, I.; et al., The rest-frame optical luminosity density,colour, and stellar mass density of the universe from $\mathrm{z}=0$ to $\mathrm{z}=3$., Astrophys. J., 2003, 599,847-864.

45. Brinchmann, J.; Ellis, R.S. The mass assembly and star formation characteristics of field galaxies of known morphology, Astrophys. J. , 2000, 536, L77-L80.

46. Elsner, F.; Feulner, G.; Hopp, U. The impact of Spitzer infrared data on stellar mass estimates. Astron. Astrophys., 2008, 477, 503-512.

47. Drory,N., Salvato,M., Gabasch,A.,et al, The stellar mass function of galxies to $z \sim 5$, Astrophysical Journal, 2005, 619, L131-L134.

48. Drory.N., Alvarez, M., The contribution of star formation and merging to stellar mass buildup in galaxies, The Astrophysical Journal, 2008, 680, 41-53.

49. Fontana, A., et. al., The assembly of massive galaxies from near Infrared observations of Hubble deepfield south, Ap. J. Lett., 2003, 594, L9-L12.

50. Fontana, A., et al., The galaxy mass function up to $z=4$ in the GOODS-MUSIC sample, Astron. Astrophys., 2006, 459, 745-757.

51. Cohen, J.G. CALTECH faint galaxy redshift survey. Astrophys. J., 2002, 567, 672-701.

52. Conselice, C.J.; Blackburne, J.A.; Papovich, C. The luminosity, stellar mass, and number density evolution of field galaxies. Astrophys. J., 2005, 620, 564-583. 
53. Borch,A.,Meisenheimer,K.,Bell,E.F.,et al.,The stellar masses of 25000 galaxies at $0.2<\mathrm{z}<1.0$ estimated by COMBO-17 survey, Astronomy and Astrophysics, 2006, 453, 869-881.

54. ESA Euclid Definition Study Report, 30 September 2011. Accessed 28 Sept. 2019, sci.esa.int/web/euclid/-/48983-euclid-definition-study-report-esa-sre-2011-12/

55. NASA WFIRST-AFTA Science Definition Team Final Report, Feb 13, 2015, Accessed 28 Sept . 2019. wfirst.gsfc.nasa.gov/science/sdt_public/WFIRST-AFTA_SDT_Report_Briefing _to_Hertz_150219_Final_RevA.pdf

56. Schlegel, D.J.; Bebek, C.; Heetderks, H.; Ho, S.; et al., The ground-based StageIV BAO Experiment, arXiv: 0904.0468v3, 2009.

57. Frieman, J.A.; Turner, M.S.; Huterer, D. Dark energy and the accelerating universe, Ann. Rev.Astron. Astrophys., 2008, 46, 385-432.

58. Guzzo, L.; Pierleoni, M.; Meneux, B.; Branchini, E.; et al., A test of the nature of cosmic acceleration using galaxy redshift distortions. Nature, 2008, 451, 541-544.

59. Devine, S., The Insights of Algorithmic Entropy, Entropy, 2009, 11, 85-110

60. Ade, P. et al.; Plank Collaboration. Planck 2013 results. XVI. Cosmological parameters, Astron. Astrophys., 2014, 571, A16.

61. Ade, P. et al.; Plank Collaboration. Planck 2015 results. XIII. Cosmological parameters, Astron. Astrophys., 2016, 594, A13.

62. Ade, P., et al.; Planck Collaboration. Planck 2015 results. XIV. Dark energy and modified gravity, Astron. Astrophys., 2016, 594, A14.

63. Planck collaboration, Planck 2018 results. VI, Cosmological Parameters, ArXiV1807.06209, 2018. Accessed 28 Sept. 2019.

64. Chevallier, M.; Polarski, D. Accelerating universes with scaling dark matter. Int. J. Mod. Phys.D, 2001, 10, 213-224.

65. Rest, A., Scolnic, D., Foley, R.J., Huber, M.E., et al., Cosmological constraints from measurements of type 1A supernovae discovered during the first 1.5 years of the Pan_STARRs1 survey, Astrophysical J., 2014, 795:44(34pp)

66. Shafer, D.L. \& Huterer, D., Chasing the phantom: A closer look at type 1 A supernovae and the dark energy equation of state, Phys. Rev. D, 2014, 89, 063510(11pp).

67. Cheng, C., \& Huang, Q-G.,Dark side of the universe after Planck data, Phys. Rev. D, 2014, 89,043003, (8 pp).

68. 68. Xia, J-Q., Li, H., Zhang, X.,Dark energy constraints after new Planck data, Phys. Rev. D, 2013, 88, $063501,8 \mathrm{p}$

69. Delubac, T., Bautista, J.E., Busca,N.G., Rich,J., et al., Baryon acoustic oscillations in the Ly $\alpha$ forest of BOSS DR11 quasars, Astronomy and Astrophysics, 2015, 574, A59 (17pp).

70. Riess,A.G., Casertano, S., Yuan,W., et al., Large Magellanic Cloud Cepheid Standards provide a 1\% foundation for determination of the Hubble Constant and stronger evidence for physics beyond $\Lambda$ CDM, Accepted by Ap. J., ArXiv 1903.07603, 27March 2019.

71. McGaugh, S.S., Lelli, F., Schomobert, J.M., The Radial Acceleration Relation in Rotationally Supported Galaxies, Phys. Rev. Lett., 2016, 117, 201101

72. Lellil, F., McGaugh,S.S., Schombert,J.M., Pawlowskil, M.S., One Law to Rule them all: the Radial Acceleration Relation of Galaxies, Astrophysical J., 2017, 836:152 (23pp)

73. Markevich,M., Gonzalez,H., Clowe,D., Vikhlinin,A., et al., Direct constraints on the dark matter self-interaction cross-section from the merging galaxy cluster 1E0657-56, Astrophys. J. , 2004, 606 (2), 819-824.

74. Harvey, D., Massey, R., Kitching, T., Taylor, A., \& Tittley, E. The non gravitational interactions of dark matter in colliding galaxy clusters. Science, 2015, 347, (62290), 1462-1465.

75. Massey, R., Williams, L., Smit, R., Swinbank, M., et al., The behaviour of dark matter associated with four bright Cluster galaxies in the 10kpc core of Abell 3827.

Mon. Not. R. Astron. Soc., 2015, 449(4), 3393-3406.

76. Viola, M., Cacciato, M., Brouwer,M., Kuijken, K.,et al., Dark matter halo properties of GAMA galaxy groups from 100 square degrees of KiDS weak lensing data, Mon. Not. R. Astron. Soc., 2015, 452, 3529-3550.

77. Grillo, C., Projected central dark matter fractions and densities in massive early-type galaxies from the Sloan Digital Sky Survey, Astrophysics Journal, 2010, 722, 779-787. 
78. Stott,J.P., Swinbank,A.M., Johnson,H.L., et al., The KMOS Redshift One Spectroscopic Survey (KROSS): Dynamical properties, gas and dark matter fractions of typical $\mathrm{z} \sim 1$ star-forming Galaxies, Mon. Not.,R. Astron. Soc., 2016, 457, 1888-1904.

79. Genzel,R.,Forster Schreiber,N.M.,Ulber,H.,Lamg,P., et al., Strongly baryon-dominated disk galaxies at the peak of galaxy formation ten billion years ago, Nature, 2017, 543, 397-401.

80. Bland-Hawthorn, J. \& Gerhard, O. The galaxy in context: structural, kinematic, and integrated properties. Annu. Rev. Astron. Astrophys. 2016, 54, 529-596.

81. Miyatake, H., More,S., Takada, M., Spergel, D.N., et el., Evidence of Halo Assembly Bias in Massive Clusters. Phys. Rev. Lett., 2016, 116, 041301 (5pp).

82. Muller, O., Pawlowski, M.S., Jerjen,H.,Lelli,F., A whirling plane of satellite galaxies around Centaurus A challenges CDM cosmology, Science, 2018, 359, 534-537.

83. Boylan-Kolchin,M., Galaxy motions cause trouble for cosmology, Science, 2018, 359, 520-521.

84. Weinberg, S. The cosmological constant problem. Rev. Mod. Phys., 1989, 61, 1-23.

85. Peebles, P.J.E. Principles of Physical Cosmology; Princeton University Press, 1993

86. Gough, M.P., Carozzi, T., Buckley, A.M. On the similarity of Information Energy to Dark Energy. arXiv 6p, astro-ph/0603084, 2006.

87. Gough, M.P., Information Equation of State, Entropy, 2008, 10, 150-159.

88. Milgrom, M.., A modification of the Newtonian dynamics - Implications for galaxies. Astrophysical Journal, 1983, 270, 371-389.

89. Arbey, A., Cosmological constraints on unifying Dark Fluid Models, The Open Astronomy J., 2008, 1, 27-38.

90. Bull,P.,Akrami,Y.,Adamek,j.,et al., Beyond $\Lambda$ CDM: Problems, solutions, and the road ahead, Physics of the Dark Universe, 2016, 12 , 56-99, \& arXiv:1512.05356. 